\title{
Invited commentary on: Jan Dalenbäck, Christer Andersson, Bengt Anesten, Stellan Björck, Stefan Eklund, Olof Magnusson, Gunnar Rimbäck, Bo Stenquist, and Nils Wedel (2008) Prolene Hernia System, Lichtenstein mesh and plug-and-patch for primary inguinal hernia repair: 3-year outcome of a prospective randomised controlled trial
}

\author{
The BOOP study: Bi-layer and connector, On-lay, and On-lay with Plug for inguinal \\ hernia repair
}

\section{J. R. DeBord}

Received: 27 April 2008/Accepted: 10 October 2008/Published online: 24 February 2009

(C) Springer-Verlag 2009

The authors of the BOOP study (Bi-layer and connector, On-lay, and On-lay with Plug for inguinal hernia repair; doi:10.1007/s10029-008-0443-4) have provided surgeons with some very interesting and important clinical data in a very nicely structured randomized and prospective controlled study, the like of which are much easier to carry out in a relatively closed system that exists in Sweden versus the more random problems of patient follow-up in North America. The long-term follow-ups are admirable and give great credence to their data.

As I have long suspected, the purported difference in the various commonly used polypropylene-based techniques for inguinal hernia repair have been based more on marketing than on science. This study gives us the comfort to suggest that any of the techniques are adequate and are associated with a high degree of success and a low degree of complications, and that both the success and the complications are not significant between the three different commonly used techniques. While the mean operating time in this study was somewhat shorter for the plug and PHS systems versus a Lichtenstein repair, the differences were not clinically meaningful in my opinion, and while there may be more anterior dissection required for a properly performed Lichtenstein repair, there is additional preperitoneal dissection that is necessary for the plug and PHS systems which is not required for an onlay mesh repair. The

J. R. DeBord $(\bowtie)$

Division of Vascular Surgery, Department of Surgery,

University of Illinois College of Medicine at Peoria,

Peoria, IL, USA

e-mail: j.debord@peoriasurgical.com low incidence of long-term pain issues was a pleasant finding and was consistent between all of the techniques utilized. The fact that prophylactic antibodies were not administered in most cases, even though a foreign body mesh was implanted, concerns me somewhat and certainly is an issue that would be of concern to the litigious environment of North American surgery, and I believe that prophylactic antibiotics involving a preoperative dose, and possibly a postoperative dose, are likely to be routine in any mesh repair in the US and would recommend that surgeons utilize prophylactic antibiotics when a mesh is to be implanted, despite the low incidence of infectious complications in this series.

As the cost of health care continually spirals nearly out of our control, one could argue that, based on this study, the proper repair would be the one that utilizes the least expensive mesh, which, I believe, based on our regional cost analysis, would favor the Lichtenstein repair as the least expensive, utilizing the lightest weight, larger pore size polypropylene mesh. I also wonder about the necessity of permanent polypropylene sutures for inguinal hernia repair with mesh, since the tissue ingrowth of the mesh (especially lightweight large pore-size mesh) should clearly have the mesh well incorporated and secure by the time any absorbable sutures were reabsorbed and the lack of permanent polypropylene sutures will probably result in fewer long-term pain issues.

I congratulate the authors on a well designed and clinically important study that gives a number of important take-home messages for practical surgeons worldwide. 